\title{
Erratum: Absence of gyratons in the Robinson-Trautman class [Phys. Rev. D 89, 124029 (2014)]
}

\author{
Robert Švarc and Jiří Podolský
}

(Q) (Received 11 February 2019; published 25 February 2019)

DOI: 10.1103/PhysRevD.99.049901

The evaluation of equations (54) and (55) for the energy-momentum tensor is not correct in this paper. These equations should read

$$
\begin{aligned}
& T_{u p, r}+(D-2) \Theta T_{u p}=0, \\
& T_{u u, r}+(D-2) \Theta T_{u u}=g^{p q} T_{u p \| q}+g^{r p}{ }_{, r} T_{u p},
\end{aligned}
$$

so that the correct integrated forms (63) and (64) are

$$
\begin{aligned}
& T_{u p}=\mathcal{J}_{p} r^{2-D}, \\
& T_{u u}=\mathcal{N} r^{2-D}-\mathcal{J}^{p} \| p_{p} r^{1-D}+f^{p} \mathcal{J}_{p} r^{3-2 D} .
\end{aligned}
$$

Unfortunately, this mistake lead us to the incorrect statement about the nonexistence of gyratons within the RobinsonTrautman class of spacetimes, as concluded in Sec. III E. Since the overall results are now completely changed, we have published the detailed correction and Erratum, with explicit general Robinson-Trautman gyratons, in the new paper [1].

[1] J. Podolský and R. Švarc, Gyratons in the Robinson-Trautman and Kundt classes, Phys. Rev. D 99, 044004 (2019). 